\title{
Plasma microRNA biomarker detection for mild cognitive impairment using differential correlation analysis
}

\author{
Mitsunori Kayano ${ }^{1,2^{*}}$ (D), Sayuri Higaki² ${ }^{2}$ Jun-ichi Satoh ${ }^{3}$, Kenji Matsumoto ${ }^{4}$, Etsuro Matsubara ${ }^{5,6}$, \\ Osamu Takikawa ${ }^{7}$ and Shumpei Niida ${ }^{2}$
}

\begin{abstract}
Background: Mild cognitive impairment $(\mathrm{MCl})$ is an intermediate state between normal aging and dementia including Alzheimer's disease. Early detection of dementia, and $\mathrm{MCl}$, is a crucial issue in terms of secondary prevention. Blood biomarker detection is a possible way for early detection of $\mathrm{MCl}$. Although disease biomarkers are detected by, in general, using single molecular analysis such as t-test, another possible approach is based on interaction between molecules.

Results: Differential correlation analysis, which detects difference on correlation of two variables in case/control study, was carried out to plasma microRNA (miRNA) expression profiles of 30 age- and race-matched controls and 23 Japanese $\mathrm{MCl}$ patients. The 20 pairs of miRNAs, which consist of 20 miRNAs, were selected as MCI markers. Two pairs of miRNAs (hsa-miR-191 and hsa-miR-101, and hsa-miR-103 and hsa-miR-222) out of 20 attained the highest area under the curve (AUC) value of 0.962 for $\mathrm{MCl}$ detection. Other two miRNA pairs that include hsa-miR-191 and hsa-miR-125b also attained high AUC value of $\geq 0.95$. Pathway analysis was performed to the MCI markers for further understanding of biological implications. As a result, collapsed correlation on hsa-miR-191 and emerged correlation on hsa-miR-125b might have key role in $\mathrm{MCl}$ and dementia progression.
\end{abstract}

Conclusion: Differential correlation analysis, a bioinformatics tool to elucidate complicated and interdependent biological systems behind diseases, detects effective $\mathrm{MCl}$ markers that cannot be found by single molecule analysis such as t-test.

Keywords: Molecular network, Coexpression, Alzheimer's disease, Dementia

\section{Background}

Early detection of dementia is a crucial issue in terms of secondary prevention. Mild cognitive impairment (MCI) is an intermediate state between normal aging and dementia including Alzheimer's disease [1-3]. On average, more than half MCI patients convert to dementia in 5 years, but some MCI patients remain stable or recover to normal over time [3-5]. This is why early detection and treatment of MCI is incredibly important.

*Correspondence: kayano@obihiro.ac.jp

${ }^{1}$ Research Center for Global Agromedicine, Obihiro University of Agriculture and Veterinary Medicine, Obihiro, Hokkaido, Japan

${ }^{2}$ Medical Genome Center, National Center for Geriatrics and Gerontology, Obu, Aichi, Japan

Full list of author information is available at the end of the article
Blood biomarkers can be useful for early detection of MCI. The present study is based on the hypothesis that neurite and synapse destruction, which are pathologic processes characteristic of early stages of $\mathrm{AD}$, other neurodegenerative diseases, and $\mathrm{MCI}$ syndrome in general, can be detected in vitro by quantitative analysis of brainenriched cell-free microRNA (miRNA) in the blood [6]. MiRNAs, a class of endogenous small non-coding RNAs, mediate posttranscriptional regulation of protein-coding genes by binding to the 3' untranslated region of target mRNAs, leading to translational inhibition or mRNA destabilization or degradation $[7,8]$. Overall, the whole human miRNA regulates greater than $60 \%$ of all proteincoding genes [9]. Importantly, cell-free miRNA have been shown to be stable in blood samples [10], and aberrant 
Table 1 Summary of participants in our study. Sample size, mean age and mean score of mini mental state exam (MMSE) are shown

\begin{tabular}{lllll}
\hline Class & & Total & Male & Female \\
\hline Age-matched controls & \# of Participants & 30 & 12 & 18 \\
(Nornal) & Age & 70.4 & 69.3 & 71.1 \\
& MMSE & 28.6 & 28.9 & 28.4 \\
MCl patients & \# of Participants & 23 & 11 & 72 \\
& Age & 72.8 & 70.8 & 24.6 \\
\hline
\end{tabular}

regulation of miRNA plays a central role in pathological events underlying cancers and neurodegenerative diseases [11-13].

A common statistical approach to detect disease biomarkers is differential expression analysis usually based on t-test between controls and patients [14]. Serum and plasma miRNA biomarkers for $\mathrm{AD}$ have been detected by differential expression analysis $[15,16]$. Although differential expression analysis is a single molecular analysis, another possible approach is based on interaction between molecules. Such approaches, which are based on the interaction between molecules, can detect more stable and accurate biomarkers, since the interaction is array- and kit-free: a difference in mean can be easily affected by a small change in absolute expression value, but the interaction-based approach can be robust in that change. Differential correlation analysis (differential coexpression analysis, $[17,18])$, an interaction-based approach, finds different types of biomarkers in terms of correlation change between controls and patients.
Differential correlation has been observed in $\mathrm{AD}$ and cancers $[19,20]$.

In this paper, differential correlation analysis was carried out to plasma miRNA expression profiles of 30 agematched controls and 23 MCI patients in Japan. Pathway analysis was performed to the detected MCI biomarkers for further understanding of biological implications of the MCI markers.

\section{Methods}

\section{Participants}

The use of human volunteer in this study was approved by the Ethical Review Board of Japan's National Center for Geriatrics and Gerontology (NCGG) and the Committee of Medical Ethics of Hirosaki University School of Medicine Institutional Review Board in Japan. We used blood samples collected in NCGG Biobank and Hirosaki University School of Medicine and Hospital. Written informed consent was obtained from all participants or their family prior to the study. The characteristics of the

Table 285 miRNAs in this study

\begin{tabular}{|c|c|c|c|c|}
\hline hsa-let-7b & hsa-miR-142-5p & hsa-miR-186 & hsa-miR-24 & hsa-miR-374b \\
\hline hsa-let-7d* & hsa-miR-143 & hsa-miR-18a & hsa-miR-25 & hsa-miR-378 \\
\hline hsa-let-7f & hsa-miR-144 & hsa-miR-191 & hsa-miR-26a & hsa-miR-423-3p \\
\hline hsa-let-7g & hsa-miR-145 & hsa-miR-192 & hsa-miR-26b & hsa-miR-423-5p \\
\hline hsa-let-7i & hsa-miR-146a & hsa-miR-197 & hsa-miR-27a & hsa-miR-424 \\
\hline hsa-miR-101 & hsa-miR-148a & hsa-miR-1979 & hsa-miR-27b & hsa-miR-425 \\
\hline hsa-miR-103 & hsa-miR-148b & hsa-miR-199a-3p & hsa-miR-29a & hsa-miR-425* \\
\hline hsa-miR-106a & hsa-miR-150 & hsa-miR-199a-5p & hsa-miR-29c & hsa-miR-451 \\
\hline hsa-miR-107 & hsa-miR-151-3p & hsa-miR-19b & hsa-miR-30b & hsa-miR-484 \\
\hline hsa-miR-122 & hsa-miR-151-5p & hsa-miR-20a & hsa-miR-30c & hsa-miR-486-5p \\
\hline hsa-miR-125b & hsa-miR-152 & hsa-miR-21 & hsa-miR-30e & hsa-miR-505 \\
\hline hsa-miR-126 & hsa-miR-15a & hsa-miR-22 & hsa-miR-320a & hsa-miR-590-5p \\
\hline hsa-miR-126* & hsa-miR-15b & hsa-miR-221 & hsa-miR-320b & hsa-miR-652 \\
\hline hsa-miR-139-5p & hsa-miR-16 & hsa-miR-222 & hsa-miR-324-3p & hsa-miR-92a \\
\hline hsa-miR-140-3p & hsa-miR-17 & hsa-miR-223 & hsa-miR-335 & hsa-miR-93 \\
\hline hsa-miR-140-5p & hsa-miR-181a & hsa-miR-23a & hsa-miR-338-3p & hsa-miR-99a \\
\hline hsa-miR-142-3p & hsa-miR-185 & hsa-miR-23b & hsa-miR-342-3p & hsa-miR-99b \\
\hline
\end{tabular}


participants are shown in Table 1: the participants were 30 age- and race-matched controls (Normal, 12 males and 18 females, mean age of 70.4) and 23 Japanese MCI patients (11 males and 12 females, mean age of 72.8). In NCGG, amnestic MCI (MCI) was diagnosed following the criteria defined by Petersen et al. [5].

\section{Sample preparation}

Total RNA was extracted from plasma using the miRNeasy Mini Kit (Qiagen) according to the manufacturer's instructions with the following modifications. Plasma was thawed on ice and centrifuged at $3000 \times \mathrm{g}$ for $5 \mathrm{~min}$ in a $4{ }^{\circ} \mathrm{C}$ microcentrifuge. An aliquot of $200 \mu \mathrm{L}$ of plasma per sample was transferred to a new tube and $750 \mu \mathrm{L}$ of Qiazol mixture containing $1.25 \mu \mathrm{g} / \mathrm{mL}$ of MS2 bacteriophage RNA (Roche Applied Science) was added to the plasma. The tube was mixed and incubated for $5 \mathrm{~min}$ followed by the addition of $200 \mu \mathrm{L}$ chloroform. The tube was mixed, incubated for $2 \mathrm{~min}$ and centrifuged at $12,000 \times \mathrm{g}$ for $15 \mathrm{~min}$ in a $4{ }^{\circ} \mathrm{C}$ microcentrifuge. The upper aqueous phase was transferred to a new microcentrifuge tube and 1.5 volume of $100 \%$ ethanol was added. The contents were mixed thoroughly and $750 \mu \mathrm{L}$ of the sample was transferred to a Qiagen RNeasy Mini spin column in a collection tube followed by centrifugation at $15,000 \times \mathrm{g}$ for $30 \mathrm{sec}$ at room temperature. The process was repeated until all remaining sample had been loaded. The spin column was rinsed with $700 \mu \mathrm{L}$ Qiagen RWT buffer and centrifuged at $15,000 \times \mathrm{g}$ for $1 \mathrm{~min}$ at room temperature followed by another rinse with $500 \mu \mathrm{L}$ Qiagen RPE buffer and centrifuged at $15,000 \times \mathrm{g}$ for $1 \mathrm{~min}$ at room temperature. A rinse step (500 $\mu \mathrm{L}$ Qiagen RPE buffer) was repeated twice. The spin column was transferred to a new collection tube and centrifuged at $15,000 \times \mathrm{g}$ for $2 \mathrm{~min}$ at room temperature. The spin column was transferred to a new microcentrifuge tube and the lid was left open for 1 min to allow the column to dry. Total RNA was eluted by adding $50 \mu \mathrm{L}$ of RNase-free water to the membrane of the spin column and incubating for $1 \mathrm{~min}$ before centrifugation at $15,000 \times \mathrm{g}$ for $1 \mathrm{~min}$ at room temperature. The RNA was stored in a $-80{ }^{\circ} \mathrm{C}$ freezer.

\section{microRNA real-time qPCR}

For reverse transcription, $19.2 \mu \mathrm{L}$ of RNA eluate was used in total $80 \mu \mathrm{L}$ reactions with the miRCURY LNA $^{\mathrm{Tm}}$ Universal RT cDNA synthesis kit (Exiqon). The

Table 3 Summary of the 20 pairs of miRNAs detected by differential correlation between Normal and MCI. The miRNA pairs are ranked by the difference of the correlation coefficients. The mean AUC value for the 20 miRNA pairs is $0.800 \pm 0.051$

\begin{tabular}{|c|c|c|c|c|c|c|c|}
\hline Rank & Pair of miRNAs & & $\left|r_{1}-r_{2}\right|$ & $\begin{array}{l}\log _{10} \\
p \text {-value }\end{array}$ & AUC & $\begin{array}{l}\text { Correlation } \\
\text { Normal }\left(r_{1}\right)\end{array}$ & $\begin{array}{l}\text { Coefficients } \\
\mathrm{MCl}\left(r_{2}\right)\end{array}$ \\
\hline 1 & hsa-miR-191 & hsa-miR-590-5p & 0.963 & -3.76 & 0.880 & 0.764 & -0.200 \\
\hline 2 & hsa-miR-125b & hsa-miR-18a & 0.930 & -3.55 & 0.733 & -0.218 & 0.712 \\
\hline 3 & hsa-miR-140-3p & hsa-miR-191 & 0.921 & -2.85 & 0.800 & 0.540 & -0.381 \\
\hline 4 & hsa-miR-103 & hsa-miR-19b & 0.917 & -3.56 & 0.797 & 0.776 & -0.141 \\
\hline 5 & hsa-miR-192 & hsa-miR-197 & 0.912 & -3.61 & 0.867 & -0.281 & 0.631 \\
\hline 6 & hsa-miR-191 & hsa-miR-19b & 0.911 & -4.10 & 0.854 & 0.826 & -0.085 \\
\hline 7 & hsa-miR-152 & hsa-miR-191 & 0.892 & -3.42 & 0.863 & 0.772 & -0.121 \\
\hline 8 & hsa-miR-103 & hsa-miR-590-5p & 0.888 & -3.24 & 0.749 & 0.614 & -0.275 \\
\hline 9 & hsa-miR-191 & hsa-miR-320a & 0.873 & -3.38 & 0.872 & 0.691 & -0.182 \\
\hline 10 & hsa-miR-125b & hsa-miR-20a & 0.871 & -3.80 & 0.801 & -0.090 & 0.781 \\
\hline 11 & hsa-miR-106a & hsa-miR-125b & 0.869 & -3.94 & 0.785 & -0.083 & 0.786 \\
\hline 12 & hsa-miR-101 & hsa-miR-103 & 0.865 & -3.65 & 0.768 & 0.805 & -0.060 \\
\hline 13 & hsa-miR-125b & hsa-miR-24 & 0.840 & -3.42 & 0.801 & -0.073 & 0.768 \\
\hline 14 & hsa-miR-101 & hsa-miR-191 & 0.831 & -3.96 & 0.871 & 0.822 & -0.009 \\
\hline 15 & hsa-miR-103 & hsa-miR-222 & 0.828 & -3.24 & 0.745 & 0.622 & -0.207 \\
\hline 16 & hsa-miR-197 & hsa-miR-378 & 0.820 & -2.78 & 0.810 & -0.234 & 0.586 \\
\hline 17 & hsa-miR-103 & hsa-miR-223 & 0.815 & -3.79 & 0.786 & 0.840 & 0.025 \\
\hline 18 & hsa-miR-125b & hsa-miR-223 & 0.815 & -3.49 & 0.765 & -0.015 & 0.800 \\
\hline 19 & hsa-let-7b & hsa-miR-125b & 0.811 & -3.75 & 0.718 & -0.056 & 0.755 \\
\hline 20 & hsa-miR-125b & hsa-miR-484 & 0.801 & -3.52 & 0.739 & -0.078 & 0.723 \\
\hline
\end{tabular}




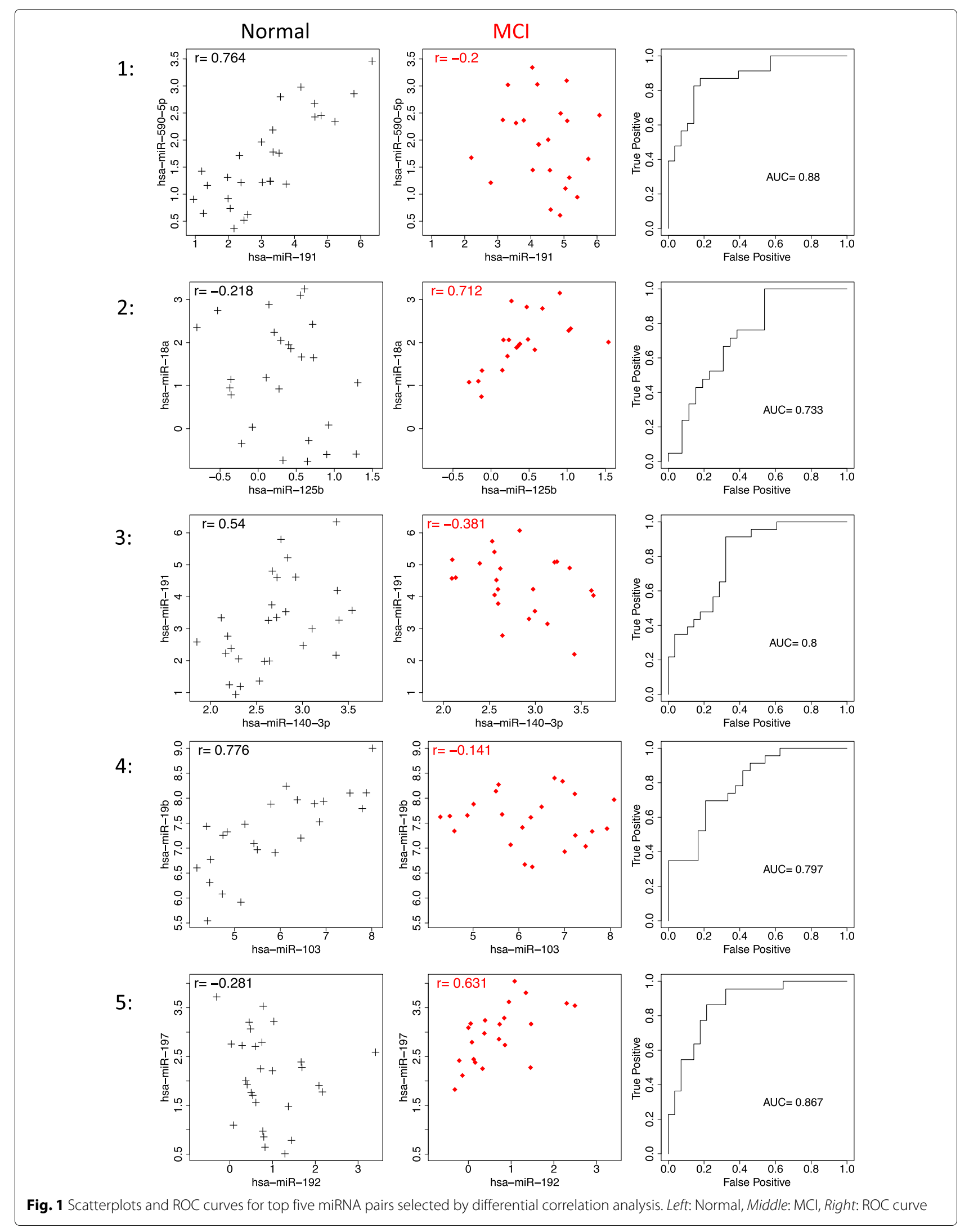


cDNA products were diluted 57.25 fold $(80 \mu \mathrm{L}$ cDNA reactions $+4500 \mu \mathrm{L}$ water) and assayed in $10 \mu \mathrm{L}$ PCR reactions according to the protocol for the miRCURY LNA ${ }^{\text {mw }}$ Universal RT microRNA PCR System; each microRNA was assayed once by qPCR on the microRNA Ready-to-Use PCR, Human panel I and panel II, V2. Negative controls excluding template from the reverse transcription reaction were assayed and profiled in the same manner of the other samples. The amplification was performed in a LightCycler 480 Real-Time PCR System
(Roche) in 384 well plates. The amplification curves were analyzed using the Roche LC software (ver. 1.5), both for determination of $\mathrm{Cp}$ (by the second derivative method) and for melting curve analysis.

\section{Data filtering}

The raw data was extracted from the Light cycler 480 software. The GenEx software (Exiqon) was used for data filtering analysis. Any assay data value must be detected below $\mathrm{Cp}<37$ or at least $3 \mathrm{Cp}$ lower than negative

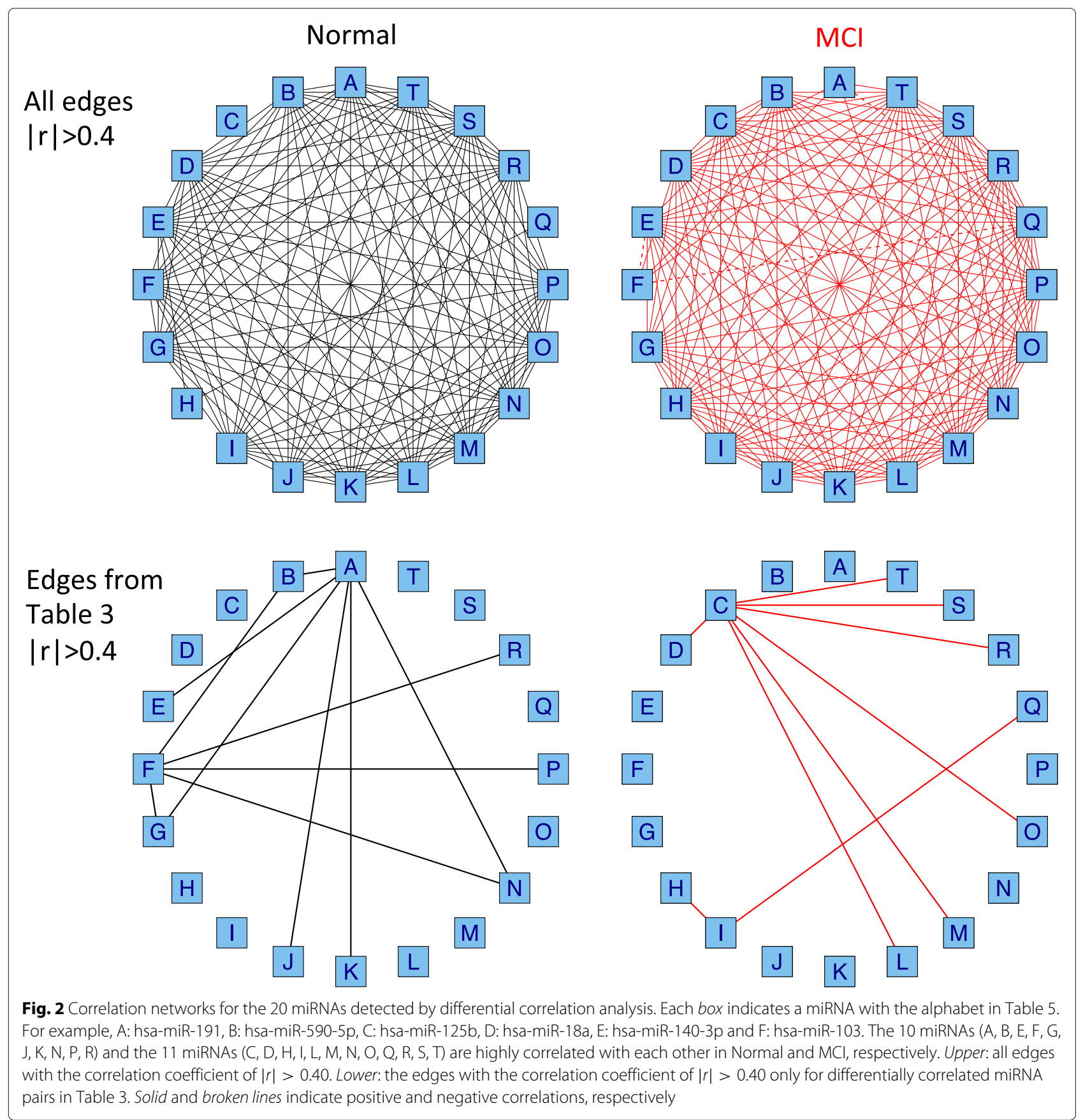


control value to be included in the data analysis. Data that did not pass these criteria were omitted from any further analysis. The amplification efficiency was calculated using the LinRegPCR software. Reactions with amplification efficiency below 1.6 were also removed. All data was normalized to the average of assays detected in each sample $(-\mathrm{dCp}=$ average $\mathrm{Cp}[<37]-$ assay $\mathrm{Cp})$. We then adopted 85 miRNAs out of 745 (Table 2) detected in over $80 \%$ samples in either one of the compared two conditions followed by filtering out low expression values $(<20 \%)$.

\section{Differential correlation analysis}

Effective MCI markers can be found by differential correlation analysis, which investigates the difference of correlation coefficients between two classes of controls and MCI patients. In differential correlation analysis in our study, all possible miRNA pairs are ranked by the difference of two correlation coefficients

$$
\left|r_{1}-r_{2}\right|
$$

where $r_{1}, r_{2}$ are Spearman's rank correlations of a miRNA pair for controls and MCI patients, respectively. MiRNA pairs with a high score of (1) are candidates of MCI markers.

Differential correlation analysis in our study also provides the $p$-value of a pair of miRNAs as a reference for statistical significance of the difference of correlation coefficients. For this purpose, normalized rank correlation $[21,22], r_{n}$, is utilized as a robust and Pearson-type correlation coefficient:

$$
r_{n}=\frac{\sum_{i} \Phi^{-1}\left\{R_{i} /(n+1)\right\} \Phi^{-1}\left\{Q_{i} /(n+1)\right\}}{\sum_{i}\left[\Phi^{-1}\{i /(n+1)\}\right]^{2}},
$$

where $\Phi$ is the distribution function of the standard normal distribution and $R_{i}$ and $Q_{i}$ are the ranks of the expression values $x_{i}$ and $y_{i}$ of two miRNAs, respectively. In our study, the value of normalized rank correlation $r_{n}$ is quite similar with that of Spearman rank correlation: the mean of the difference between normalized rank correlations and Spearman's rank correlations for all miRNA pairs was only 0.001 in our data set. Hypothesis testing to investigate the equality of two normalized rank correlation coefficients is then applied according to a likelihood ratio test in $[23,24]$. The $p$-value can be calculated through the hypothesis testing. We used Spearman's rank correlation for the difference calculation on correlation

Table 4 Summary of the top 10 two-pairs of miRNAs out of the 20 miRNA pairs detected by differential correlation analysis in Table 3.

\begin{tabular}{|c|c|c|c|c|c|c|c|}
\hline Rank & AUC & $\begin{array}{l}\text { Original } \\
\text { Rank* }\end{array}$ & $\begin{array}{l}\text { Original } \\
\text { AUC }^{*}\end{array}$ & Two-pairs of miRNAs & & $\begin{array}{l}\text { Correlation } \\
\text { Normal }\left(r_{1}\right)\end{array}$ & $\begin{array}{r}\text { Coefficients } \\
\qquad \mathrm{MCl}\left(r_{2}\right)\end{array}$ \\
\hline \multirow[t]{2}{*}{1} & 0.962 & 14 & 0.871 & hsa-miR-101 & hsa-miR-191 & 0.822 & -0.009 \\
\hline & & 15 & 0.745 & hsa-miR-103 & hsa-miR-222 & 0.622 & -0.207 \\
\hline \multirow[t]{2}{*}{2} & 0.959 & 5 & 0.867 & hsa-miR-192 & hsa-miR-197 & -0.281 & 0.631 \\
\hline & & 14 & 0.871 & hsa-miR-101 & hsa-miR-191 & 0.822 & -0.009 \\
\hline \multirow[t]{2}{*}{3} & 0.958 & 6 & 0.854 & hsa-miR-191 & hsa-miR-19b & 0.826 & -0.085 \\
\hline & & 17 & 0.786 & hsa-miR-103 & hsa-miR-223 & 0.840 & 0.025 \\
\hline \multirow[t]{2}{*}{4} & 0.957 & 1 & 0.880 & hsa-miR-191 & hsa-miR-590-5p & 0.764 & -0.200 \\
\hline & & 17 & 0.786 & hsa-miR-103 & hsa-miR-223 & 0.840 & 0.025 \\
\hline \multirow[t]{2}{*}{5} & 0.957 & 14 & 0.871 & hsa-miR-101 & hsa-miR-191 & 0.822 & -0.009 \\
\hline & & 16 & 0.810 & hsa-miR-197 & hsa-miR-378 & -0.234 & 0.586 \\
\hline \multirow[t]{2}{*}{6} & 0.952 & 12 & 0.768 & hsa-miR-101 & hsa-miR-103 & 0.805 & -0.060 \\
\hline & & 13 & 0.801 & hsa-miR-125b & hsa-miR-24 & -0.073 & 0.768 \\
\hline \multirow[t]{2}{*}{7} & 0.951 & 5 & 0.867 & hsa-miR-192 & hsa-miR-197 & -0.281 & 0.631 \\
\hline & & 9 & 0.872 & hsa-miR-191 & hsa-miR-320a & 0.691 & -0.182 \\
\hline \multirow[t]{2}{*}{8} & 0.951 & 14 & 0.871 & hsa-miR-101 & hsa-miR-191 & 0.822 & -0.009 \\
\hline & & 17 & 0.786 & hsa-miR-103 & hsa-miR-223 & 0.840 & 0.025 \\
\hline \multirow[t]{2}{*}{9} & 0.951 & 1 & 0.880 & hsa-miR-191 & hsa-miR-590-5p & 0.764 & -0.200 \\
\hline & & 15 & 0.745 & hsa-miR-103 & hsa-miR-222 & 0.622 & -0.207 \\
\hline \multirow[t]{2}{*}{10} & 0.947 & 4 & 0.797 & hsa-miR-103 & hsa-miR-19b & 0.776 & -0.141 \\
\hline & & 14 & 0.871 & hsa-miR-101 & hsa-miR-191 & 0.822 & -0.009 \\
\hline
\end{tabular}
The two-pairs of miRNAs are ranked by AUC value

*: Original rank and AUC of each pair of miRNAs in Table 3. Bold: top five miRNAs 
coefficients (1) and used the normalized rank correlation for $p$-value calculation.

Evaluation of the performance of a miRNA pair as MCI marker is not straight-forward. We here apply receiveroperator characteristic (ROC) analysis on logistic regression with an interaction term of two miRNAs:

$$
\log \frac{p}{1-p}=\beta_{0}+\beta_{1} X_{1}+\beta_{2} X_{2}+\beta_{12} X_{1} X_{2}
$$

where $p$ is the probability that a sample is in MCI class, $\beta_{0}, \beta_{1}, \beta_{2}, \beta_{12}$ are regression coefficients and $X_{1}, X_{2}$ are the expression value of two miRNAs, respectively. The interaction term $\beta_{12} X_{1} X_{2}$ is essential for the evaluation of two miRNAs detected by differential correlation analysis. If the correlation coefficient between $X_{1}$ and $X_{2}$ is altered between controls and MCI class, then the interaction term significantly affects the discrimination of $\mathrm{MCI}$ from controls. The area under the curve (AUC) value (=0 to 1$)$ is estimated through ROC analysis based on the estimated probabilities $\hat{p}_{1}, \ldots ., \hat{p}_{n}$ for all samples of controls and MCI patients. If the estimated probabilities for controls and MCI patients are much different (e.g., $\hat{p}<0.5$ for controls and $\hat{p}>0.5$ for MCI patients), then AUC value will be 1 (completely separated). In order to evaluate of the performance of several pairs of miRNAs as MCI markers, logistic regression with multiple interaction terms can be available:

$$
\begin{aligned}
\log \frac{p}{1-p}= & \beta_{0}+\beta_{1} X_{1}+\beta_{2} X_{2}+\ldots \\
& +\beta_{k} X_{k}+\sum_{(i, j) \in C} \beta_{i j} X_{i} X_{j}
\end{aligned}
$$

where $C$ is a set of miRNA pairs that are differentially correlated between controls and MCI patients. For example, four miRNA pairs (miRNA 1-2, 1-3, 3-4 and 4-5) with five miRNAs can be incorporated in the logistic regression model, $\log p /(1-p)=\beta_{0}+\beta_{1} X_{1}+\beta_{2} X_{2}+$ $\beta_{3} X_{3}+\beta_{4} X_{4}+\beta_{5} X_{5}+\beta_{12} X_{1} X_{2}+\beta_{13} X_{1} X_{3}+\beta_{34} X_{3} X_{4}+$ $\beta_{45} X_{4} X_{5}$, where $C=\{(1,2),(1,3),(3,4),(4,5)\}$ in the interaction terms $\sum_{(i, j) \in C} \beta_{i j} X_{i} X_{j}$. ROC analysis evaluates the performance of the five miRNAs as MCI markers simultaneously.

\section{Results}

\section{Differential correlation analysis}

Differential correlation analysis was applied to the data set with 85 miRNAs for age-matched samples of 30 controls and $23 \mathrm{MCI}$ patients (Tables 1 and 2). The 3570 possib le pairs from the 85 miRNAs were ranked, according to the difference of correlation coefficients between controls and MCI patients. The 20 pairs of miRNAs, which had the difference of correlation coefficients of $\left|r_{1}-r_{2}\right|>0.8$, were selected as biomarkers that distinguish MCI patients from controls (Table 3). The AUC value by each of the 20 miRNA pairs was $0.800 \pm 0.051$ ranged between 0.718 and 0.880. Figure 1 shows scatterplots and ROC curves for each of top five miRNA pairs selected by differential correlation between normal and MCI (see also Additional file 1 for the remained miRNA pairs). Figure 2 shows correlation networks of the 20 miRNA pairs.

AUC value for all two-pairs of the 20 miRNA pairs was also calculated by using (4). Table 4 shows summary of the top 10 two-pairs of miRNAs out of 190 possible pairs. Two miRNA pairs (hsa-miR-191 and hsamiR-101, and hsa-miR-103 and hsa-miR-222) attained the highest AUC value of 0.962 for MCI detection (Fig. 3). Other two miRNA pairs that include hsa-miR-191 and hsa-miR-125b also attained high AUC value of $\geq 0.95$ (Table 4).

\section{Pathway analysis}

We performed Ingenuity Pathway Analysis (IPA) about correlation networks to be lost and emerged in the MCI. Figures 4 and 5 show estimated networks through IPA on the 10 and 11 miRNAs, which are highly correlated with each other in Normal and MCI respectively. IPA revealed that the 10 highly correlated miRNAs in Normal were composed of networks surrounding Akt, IGF1, PPARA, IL6 and AGO2 genes. The IPA showed that TP53 genes directly regulated all of 11 highly correlated miRNAs in MCI. Pathways enriched for target genes of 10/11 highly

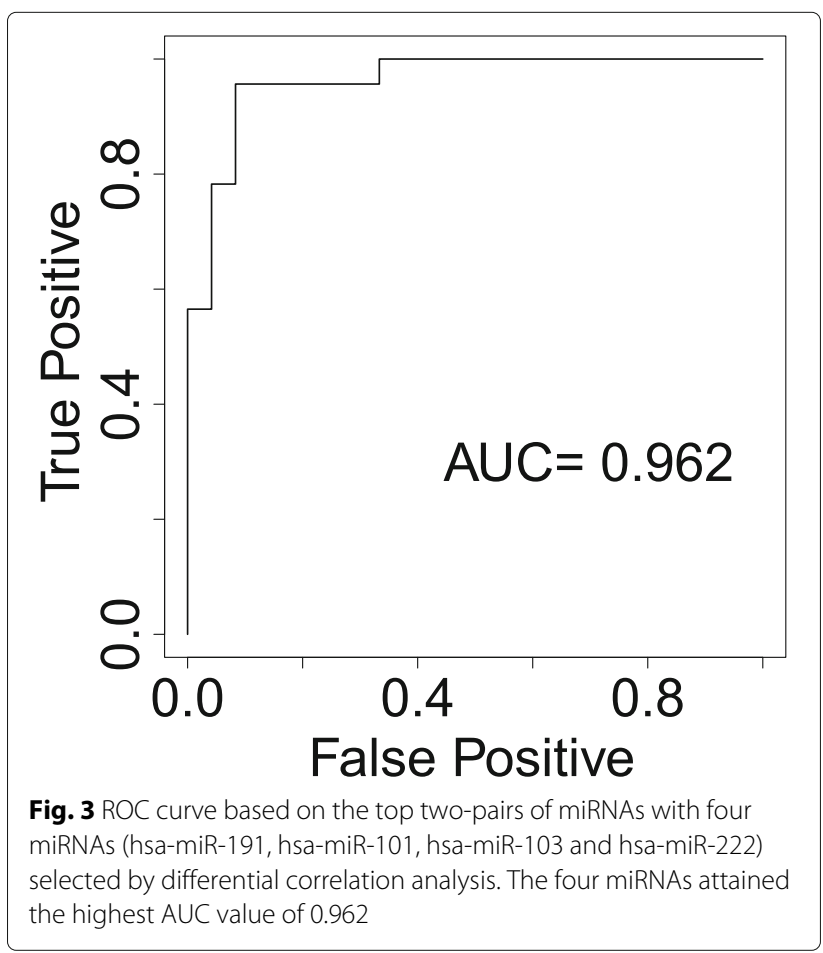




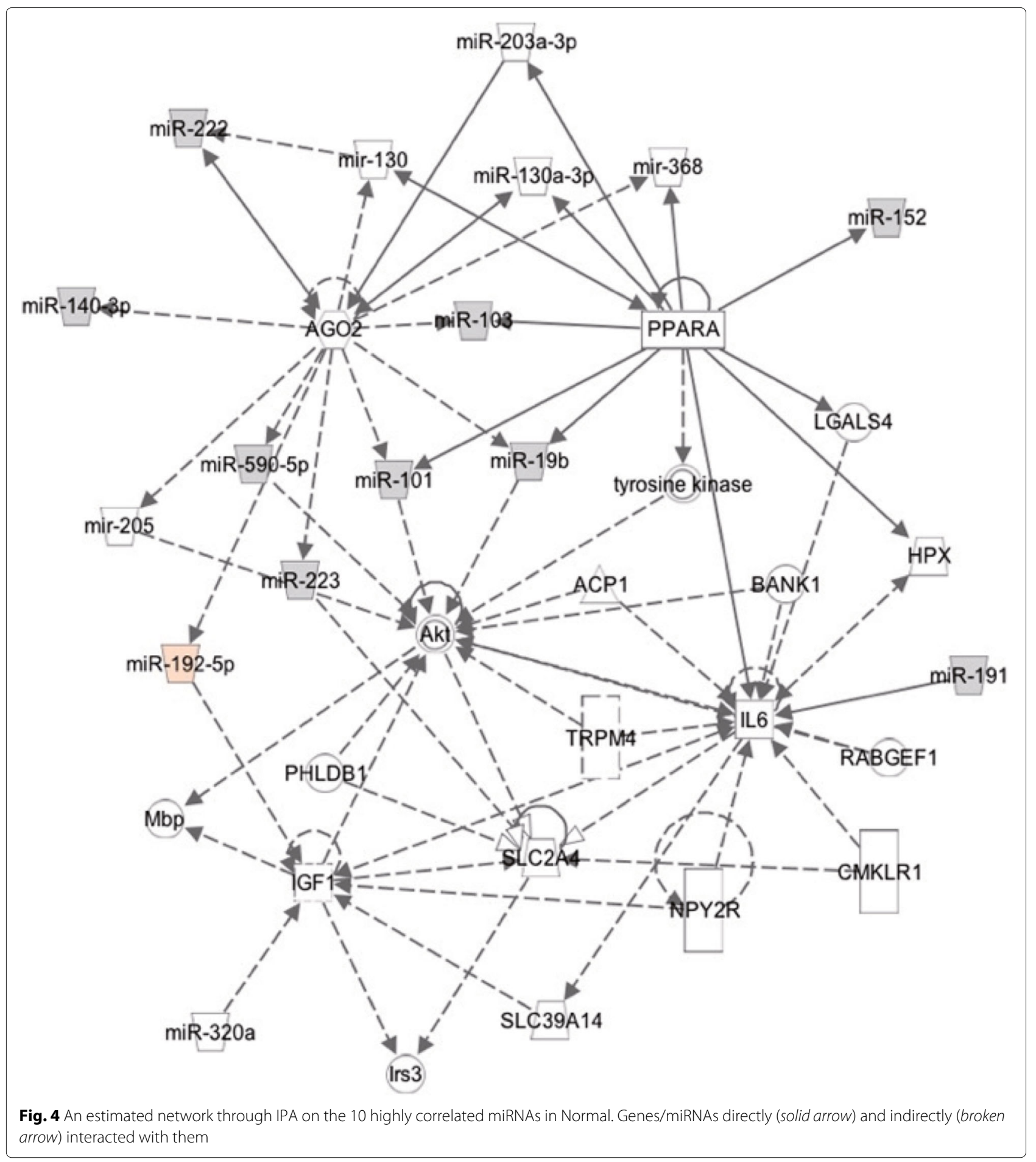

correlated miRNAs in Normal/MCI are shown in Tables 6 and 7.

\section{T-test}

Traditional t-test was applied to the same data set with 85 miRNAs for age-matched samples of 30 controls and 23
MCI patients (Tables 1 and 2). The detail was described in Additional file 2. The 22 miRNAs out of 85 were detected as MCI markers (Table 5 and Additional file 2). The AUC value by each of the 22 miRNAs was $0.784 \pm$ 0.017 ranged between 0.748 and 0.828 . Importantly, differential correlation analysis detected much different and 


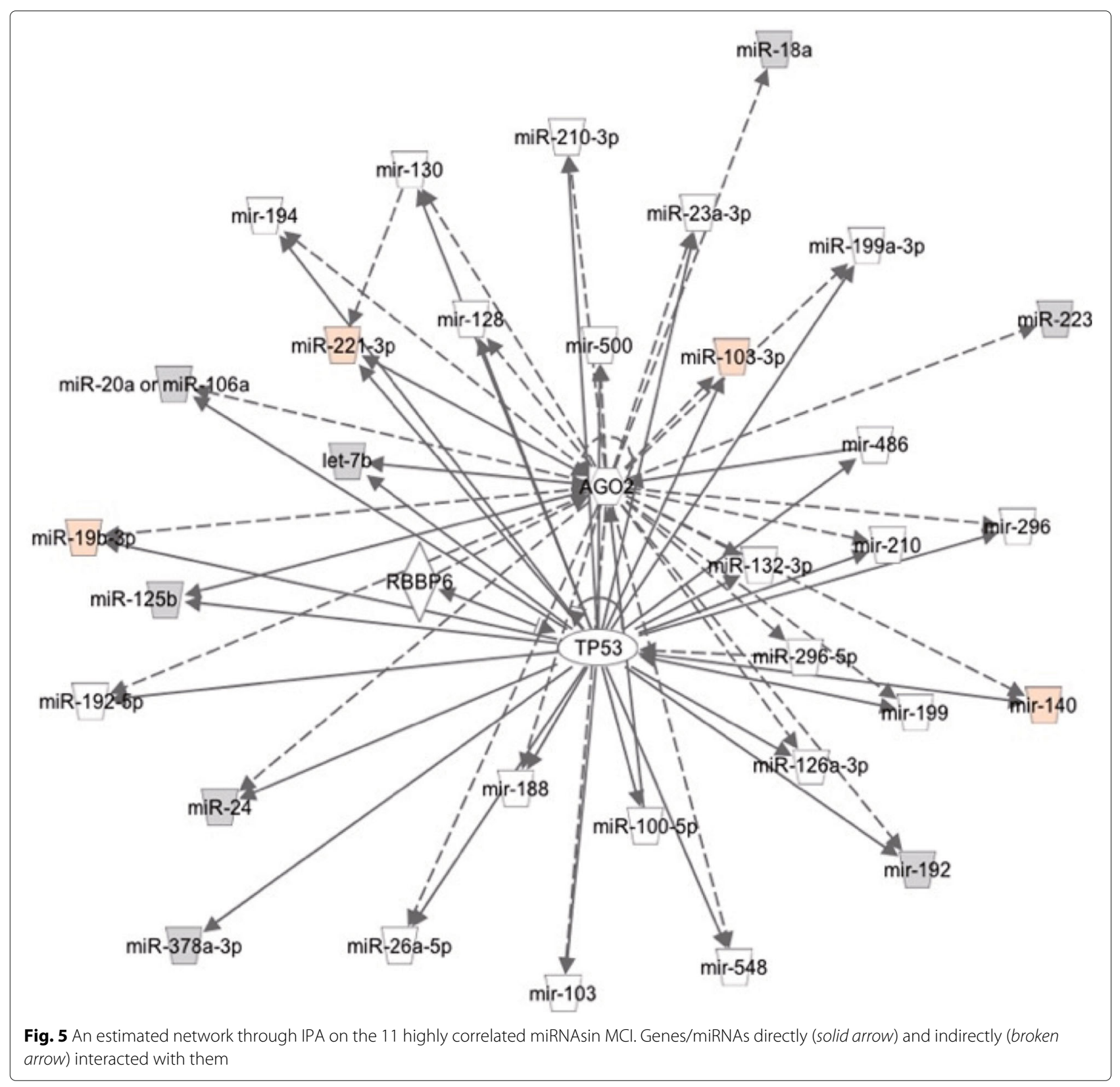

more sensitive MCI markers compared to t-test (Table 5): mean AUC value $=0.800 \pm 0.051$ (differential correlation analysis),$=0.784 \pm 0.017$ (t-test). Also, the highest AUC value of any four miRNAs from the 22 miRNAs (Figure 4 in Additional file 2) was less than the highest in the twopair approach, which investigate the performance of three to four miRNAs simultaneously, in differential correlation analysis.

\section{Discussion}

Pathway analysis, IPA, allows us for further understanding of biological implications of the detected $20 \mathrm{MCI}$ maker pairs of miRNA. Validation study and brain-based previous studies can support the results of differential correlation analysis and IPA.

IPA showed that 10 highly correlated miRNAs in Normal were composed of networks surrounding Akt, IGF1, PPARA, IL6 and AGO2 genes (Fig. 4). Akt, IGF1 and Irs3 are key molecules in insulin signaling pathway and PPARA is a regulator of lipid metabolism. Moreover, insulin, mTOR and PI3K-Akt signaling pathway were ranked among top 5 analyzed by DIANA-miRPath, which predicted miRNA targets through DIANA-microT-CDS and combined their interactions into KEGG pathway 
Table 5 The two sets of miRNAs detected by Left: differential correlation analysis and Right: t-test

\begin{tabular}{|c|c|c|c|c|c|}
\hline \multicolumn{3}{|c|}{$\begin{array}{l}\text { Differential correlation } \\
20 \text { miRNAs }\end{array}$} & \multirow[b]{2}{*}{ hsa-miR-20a } & \multicolumn{2}{|l|}{$\begin{array}{l}\text { t-test } \\
22 \text { miRNAs }\end{array}$} \\
\hline A: & hsa-miR-191 & $\mathrm{L}:$ & & hsa-miR-151-3p & hsa-miR-15b \\
\hline B: & hsa-miR-590-5p & M: & hsa-miR-106a & hsa-miR-126* & hsa-let-7d* \\
\hline C: & hsa-miR-125b & $\mathrm{N}:$ & hsa-miR-101 & hsa-miR-23a & hsa-miR-197 \\
\hline D: & hsa-miR-18a & O: & hsa-miR-24 & hsa-miR-27b & hsa-miR-30b \\
\hline E: & hsa-miR-140-3p & $P:$ & hsa-miR-222 & hsa-miR-146a & hsa-miR-185 \\
\hline$F:$ & hsa-miR-103 & Q: & hsa-miR-378 & hsa-miR-30c & hsa-miR-191 \\
\hline G: & hsa-miR-19b & $\mathrm{R}:$ & $\underline{\text { hsa-miR-223 }}$ & hsa-miR-151-5p & hsa-miR-26b \\
\hline $\mathrm{H}:$ & hsa-miR-192 & S: & hsa-let-7b & hsa-miR-23b & hsa-miR-223 \\
\hline I: & hsa-miR-197 & $\mathrm{T}:$ & hsa-miR-484 & hsa-miR-92a & hsa-miR-26a \\
\hline J: & hsa-miR-152 & & & $\underline{\text { hsa-miR-24 }}$ & hsa-miR-16 \\
\hline $\mathrm{K}:$ & hsa-miR-320a & & & hsa-miR-144 & hsa-let-7f \\
\hline
\end{tabular}

Bold: top five miRNAs in each analysis

Underline: same miRNAs in left and right sides

The alphabets in the left side are utilized in Fig. 2

Table 6 Pathways enriched for target genes of 10 highly correlated miRNAs in Normal

\begin{tabular}{llll}
\hline \# KEGG pathway & -value & \#genes & \#miRNAs \\
\hline 1 Pathways in cancer (hsa05200) & $2.13 \times 10^{-19}$ & 101 & 9 \\
2 Prostate cancer (hsa05215) & $3.39 \times 10^{-17}$ & 35 & 9 \\
3 Pl3K-Akt signaling pathway (hsa04151) & $1.92 \times 10^{-15}$ & 94 & 9 \\
4 mTOR signaling pathway (hsa04150) & $6.11 \times 10^{-14}$ & 27 & 9 \\
5 Insulin signaling pathway (hsa04910) & $1.18 \times 10^{-13}$ & 46 & 9 \\
6 Endometrial cancer (hsa05213) & $4.34 \times 10^{-13}$ & 22 & 10 \\
7 Ubiquitin mediated proteolysis (hsa04120) & $6.03 \times 10^{-13}$ & 46 & 9 \\
8 Focal adhesion (hsa04510) & $5.48 \times 10^{-12}$ & 60 & 8 \\
9 Non-small cell lung cancer (hsa05223) & $5.16 \times 10^{-10}$ & 21 & 7 \\
10 Hedgehog signaling pathway (hsa04340) & $6.68 \times 10^{-10}$ & 20 & 9 \\
\hline
\end{tabular}

Table 7 Pathways enriched for target genes of 11 highly correlated miRNAs in $\mathrm{MCl}$

\begin{tabular}{llll}
\hline \# KEGG pathway & $p$-value & \#genes & \#miRNAs \\
\hline 1 MAPK signaling pathway (hsa04010) & $1.81 \times 10^{-13}$ & 79 & 11 \\
2 Endocytosis (hsa04144) & $5.43 \times 10^{-12}$ & 63 & 10 \\
3 TGF-beta signaling pathway (hsa04350) & $5.43 \times 10^{-12}$ & 31 & 10 \\
4 Pl3K-Akt signaling pathway (hsa04151) & $3.88 \times 10^{-10}$ & 91 & 11 \\
5 Pathways in cancer (hsa05200) & $4.62 \times 10^{-10}$ & 38 & 11 \\
6 Neurotrophin signaling pathway (hsa04722) & $5.66 \times 10^{-8}$ & 30 & 11 \\
7 Prostate cancer (hsa05215) & $6.03 \times 10^{-8}$ & 41 & 10 \\
8 Ubiquitin mediated proteolysis (hsa04120) & $1.37 \times 10^{-7}$ & 29 & 11 \\
9 ErbB signaling pathway (hsa04012) & $5.11 \times 10^{-7}$ & 41 & 11 \\
10 Hepatitis B (hsa05161) & $6.96 \times 10^{-7}$ & & \\
\hline
\end{tabular}


(Table 6). These pathways included target genes of 9 miRNAs except for miR-191. Previous studies consistently reported that identified biomarkers, changed genes and networks in $\mathrm{AD}$ patients or $\mathrm{AD}$ model were involved in insulin-related signaling $[8,25,26]$. Indeed, experimentally validated evidences support key role of miR-103a-3p, miR-320a and miR-590-5p in metabolic pathway $[27,28]$ and miR-103a-3p association with $A D$ [29-31]. In Fig. 2, we found that miR-103a-3p and miR-191 served as hub miRNAs of 12 edges of pair correlations in Normal. miR-191 is also a widely used biomarker for diseases like cancers, type-2 diabetes and AD [32]. Considering the significant upregulation of miR-191 in MCI (t-test), these findings supposed that MCI stage lost miRNA correlations as cause and/or effect of changed expression balance among miR-191 and members in insulin related signaling. Lost of their correlation could become a discriminative marker for MCI.

There are newly emerged correlation network with a hub miRNA, miR-125b in MCI patient plasma. The IPA showed that TP53 genes directly regulated all of 11 highly correlated miRNAs in MCI (Fig. 5). TP53 has been explored originally as a tumor suppressor, but recently reported about other aspects to control diseases such as aging and metabolism [33]. There are accumulated studies that the change of TP53 protein, its modification and conformation were observed in AD patient brains [34-36] and blood [37]. Intriguingly, Le et al. demonstrated that miR-125b bound to 3' untranslated region of TP53 mRNA and worked as a negative regulator of TP53 [38], which means a possible presence of negative feedback loop. The result of DIANA-miRPath indicated that MAPK, TGF-beta and Neurotrophin signaling pathway were characteristic in MCI, although there were overlapped pathways in Normal and MCI (Table 7). Similarly to TP53 signaling, these pathways have common biological functions such as cell survival, cell cycle and apoptosis. In this study, change of TP53 function might be detected as generated new correlations of the downstream miRNAs.

This study focuses on biomarker detection for MCI, not on mechanism that how were plasma miRNAs produced from brain. However, brain-based studies also support reliability of hsa-miR-191 and hsa-125b as MCI markers. For example, expression change of miR-191 is required for maintenance of spine restructuring in mouse hippocampus [39], and miR-125b effects on dendritic spine morphology and synaptic physiology in hippocampal neurons of mouse [40], where it has been shown that $\mathrm{MCI}$ and $\mathrm{AD}$ is a synaptic failure [41-43].

In summary, collapsed correlation on hsa-miR-191 and emerged correlation on hsa-miR-125b might have key role in $\mathrm{MCI}$, and dementia progression.

\section{Conclusions}

Differential correlation analysis, which detects difference of correlation in case/control study, was carried out to plasma miRNA expression profiles of 30 age- and racematched controls and 23 Japanese MCI patients. The 20 miRNA pairs were selected as biomarkers for MCI. The 20 miRNAs were more sensitive and different from that by t-test.

Pathway analysis showed that, in particular, collapsed correlation on hsa-miR-191 and emerged correlation on hsa-miR-125b might have key role in MCI, and dementia progression. Differential correlation analysis detects effective MCI markers that cannot be found by single molecule analysis such as t-test. Also, differential correlation analysis could be a key bioinformatics tool to find sensitive biomarkers and to elucidate complicated biological systems behind diseases.

\section{Additional files}

Additional file 1: Supplement A. Scatterplots and ROC curves for each of top 20 pairs of miRNAs selected by differential correlation analysis between Normal and $\mathrm{MCl}$. (PDF $63.2 \mathrm{~kb}$ )

Additional file 2: Supplement B. Details of t-test and the results. The results include boxplots and $\mathrm{ROC}$ curves based on each of 22 miRNAs selected by t-test between Normal and MCl. (PDF 55.1 kb)

\section{Abbreviations}

AD: Alzheimer's disease; AUC: Area under the (ROC) curve; IPA: Ingenuity pathway analysis; MCI: Mild cognitive impairment; miRNA: MicroRNA; MMSE: Mini mental state exam; NCGG: National center for geriatrics and gerontology; ROC: Receiver-operator characteristic

\section{Acknowledgements}

Not applicable.

\section{Funding}

This work has been supported in part by the Program for Promotion of Fundamental Studies in Health Sciences conducted from the National Institute of Biomedical Innovation of Japan (10-43 and 10-44), the Research Funding for Longevity Sciences from National Center for Geriatrics and Gerontology, Japan (23-9 and 26-20), and KAKENHI \#24700290 from Japan Society for the Promotion of Science.

\section{Availability of data and materials}

Data will be available from NCGG Biobank (Medical Genome Center, http:// www.ncgg.go.jp/mgc/index.html [In preparing to upload the data]). If you have any problems to access the web site, please contact author.

\section{Authors' contributions}

MK, SH, KM, EM, OT and SN designed the study. MK, SH, KM and EM performed the analysis. MK and SH wrote the manuscript. JS, OT and SN contributed to critical review of the manuscript. All authors read and approved the final manuscript.

\section{Competing interests}

The authors declare that they have no competing interests.

\section{Consent for publication}

Not applicable.

\section{Ethics approval and consent to participate}

The use of human volunteer in this study was approved by the Ethical Review Board of Japan's National Center for Geriatrics and Gerontology (NCGG) and the Committee of Medical Ethics of Hirosaki University School of Medicine 
Institutional Review Board in Japan: reference number of 443-6 (Approved 26 February 2015), 2008-116 (Approved 28 November 2008). We used blood samples collected in NCGG Biobank and Hirosaki University School of Medicine and Hospital. Written informed consent was obtained from all participants or their family prior to the study.

\section{Author details}

${ }^{1}$ Research Center for Global Agromedicine, Obihiro University of Agriculture and Veterinary Medicine, Obihiro, Hokkaido, Japan. ${ }^{2}$ Medical Genome Center, National Center for Geriatrics and Gerontology, Obu, Aichi, Japan. ${ }^{3}$ Department of Bioinformatics and Molecular Neuropathology, Meiji Pharmaceutical University, Kiyose, Tokyo, Japan. ${ }^{4}$ Department of Allergy and Clinical Immunology, National Center for Child Health and Development, Setagaya, Tokyo, Japan. ${ }^{5}$ Department of Neurology, Hirosaki University Graduate School of Medicine, Hirosaki, Aomori, Japan. ${ }^{6}$ Department of Neurology, Oita University Faculty of Medicine, Yufu, Oita, Japan. ${ }^{7}$ Innovation Center for Clinical Research, National Center for Geriatrics and Gerontology, Obu, Aichi, Japan.

\section{Received: 27 September 2016 Accepted: 22 November 2016} Published online: 12 December 2016

\section{References}

1. DeCarli C. Mild cognitive impairment: prevalence, prognosis, aetiology, and treatment. Lancet Neurol. 2003;2(1):15-21.

2. Markesbery WR. Neuropathologic alterations in mild cognitive impairment: a review. J Alzheimers Dis: JAD. 2010;19(1):221.

3. Apostolova LG, Thompson PM, Green AE, Hwang KS, Zoumalan C, Jack CR, et al. 3D comparison of low, intermediate, and advanced hippocampal atrophy in MCl. Hum Brain Mapp. 2010;31(5):786-97.

4. Gauthier S, Reisberg B, Zaudig M, Petersen RC, Ritchie K, Broich K, et al. Mild cognitive impairment. Lancet. 2006;367(9518):1262-70.

5. Petersen RC, Doody R, Kurz A, Mohs RC, Morris JC, Rabins PV, et al. Current concepts in mild cognitive impairment. Arch Neurol. 2001;58(12): 1985-92.

6. Sheinerman KS, Tsivinsky VG, Crawford F, Mullan MJ, Abdullah L, Umansky SR. Plasma microRNA biomarkers for detection of mild cognitive impairment. Aging (Albany NY). 2012;4(9):590.

7. Guo H, Ingolia NT, Weissman JS, Bartel DP. Mammalian microRNAs predominantly act to decrease target mRNA levels. Nature. 2010;466(7308):835-40.

8. Satoh Ji, Kino Y, Niida S. MicroRNA-Seq Data Analysis Pipeline to Identify Blood Biomarkers for Alzheimer's Disease from Public Data. Biomark Insights. 2015;10:21.

9. Friedman RC, Farh KKH, Burge CB, Bartel DP. Most mammalian mRNAs are conserved targets of microRNAs. Genome Res. 2009;19(1):92-105.

10. Mitchell PS, Parkin RK, Kroh EM, Fritz BR, Wyman SK, PogosovaAgadjanyan EL, et al. Circulating microRNAs as stable blood-based markers for cancer detection. Proc Natl Acad Sci. 2008;105(30):10513-8.

11. Keller $A$, Leidinger $P$, Bauer $A$, ElSharawy A, Haas J, Backes $C$, et al. Toward the blood-borne miRNome of human diseases. Nat Methods. 2011;8(10):841-3.

12. Satoh Ji. Molecular network analysis of human microRNA targetome: from cancers to Alzheimer's disease. BioData Min. 2012;5(1):17.

13. Hayes J, Peruzzi PP, Lawler S. MicroRNAs in cancer: biomarkers, functions and therapy. Trends Mol Med. 2014;20(8):460-9.

14. Cui $X$, Churchill GA, et al. Statistical tests for differential expression in cDNA microarray experiments. Genome Biol. 2003;4(4):210.

15. Kumar P, Dezso Z, Mackenzie C, Oestreicher J, Agoulnik S, Byrne M, et al. Circulating miRNA biomarkers for Alzheimer's disease. PloS one. 2013;8(7):e69807.

16. Tan L, Yu JT, Liu QY, Tan MS, Zhang W, Hu N, et al. Circulating miR-125b as a biomarker of Alzheimer's disease. J Neurol Sci. 2014;336(1):52-6.

17. de la Fuente A. From 'differential expression'to 'differential networking'-identification of dysfunctional regulatory networks in diseases. Trends Genet. 2010;26(7):326-33.

18. Kayano M, Shiga M, Mamitsuka H. Detecting differentially coexpressed genes from labeled expression data: a brief review. Comput Biol Bioinforma IEEE/ACM Trans. 2014;11(1):154-67.

19. Choi Y, Kendziorski C. Statistical methods for gene set co-expression analysis. Bioinformatics. 2009;25(21):2780-6.
20. Amar D, Safer H, Shamir R. Dissection of regulatory networks that are altered in disease via differential co-expression. PLoS Comput Biol. 2013;9(3):e1002955.

21. Yanagawa T, Kobayashi Y, Nagayama J. Assessing the joint effects of chlorinated dioxins, some pesticides and polychlorinated biphenyls on thyroid hormone status in Japanese breast-fed infants. Environmetrics. 2003;14(2):121-8.

22. Kayano M, Imoto S, Yamaguchi R, Miyano S. Multi-omics Approach for Estimating Metabolic Networks Using Low-Order Partial Correlations. J Comput Biol. 2013;20(8):571-82.

23. Paul S. Test for the equality of several correlation coefficients. Can J Stat. 1989217-27.

24. Kayano M, Takigawa I, Shiga M, Tsuda K, Mamitsuka H. ROS-DET: robust detector of switching mechanisms in gene expression. Nucleic Acids Res. 2011;39(11):e74-e74.

25. Liang D, Han G, Feng X, Sun J, Duan Y, Lei H. Concerted perturbation observed in a hub network in Alzheimer's disease. PLoS One. 2012;7(7): e40498.

26. Jackson HM, Soto I, Graham LC, Carter GW, Howell GR. Clustering of transcriptional profiles identifies changes to insulin signaling as an early event in a mouse model of Alzheimer's disease. BMC Genomics. 2013;14(1):831.

27. Karolina DS, Tavintharan S, Armugam A, Sepramaniam S, Pek SLT, Wong MT, et al. Circulating miRNA profiles in patients with metabolic syndrome. J Clin Endocrinol Metab. 2012;97(12):E2271-E6.

28. Williams MD, Mitchell GM. MicroRNAs in insulin resistance and obesity. Exp Diabetes Res. 2012;484696.

29. Wang WX, Rajeev BW, Stromberg AJ, Ren N, Tang G, Huang Q, et al. The expression of microRNA miR-107 decreases early in Alzheimer's disease and may accelerate disease progression through regulation of $\beta$-site amyloid precursor protein-cleaving enzyme 1. J Neurosci. 2008;28(5): 1213-23.

30. Yao J, Hennessey T, Flynt A, Lai E, Beal MF, Lin MT. MicroRNA-related cofilin abnormality in Alzheimer's disease. PloS One. 2010;5(12):e15546.

31. Moncini S, Salvi A, Zuccotti P, Viero G, Quattrone A, Barlati S, et al. The role of miR-103 and miR-107 in regulation of CDK5R1 expression and in cellular migration. PloS One. 2011;6(5):e20038.

32. Nagpal N, Kulshreshtha R. miR-191: an emerging player in disease biology. Front Genet. 2014;5:99.

33. Vousden KH. Lane DP. p53 in health and disease. Nature Reviews Molecular Cell Biology. 2007;8(4):275-283.

34. Kitamura $Y$, Shimohama S, Kamoshima W, Matsuoka $Y$, Nomura $Y$, Taniguchi T. Changes of p53 in the brains of patients with Alzheimer's disease. Biochem Biophys Res Commun. 1997;232(2):418-21.

35. Di Domenico F, Cenini G, Sultana R, Perluigi M, Uberti D, Memo M, et al. Glutathionylation of the pro-apoptotic protein p53 in Alzheimer's disease brain: implications for AD pathogenesis. Neurochem Res. 2009;34(4): 727-33.

36. Stanga S, Lanni C, Govoni S, Uberti D, D'Orazi G, Racchi M. Unfolded p53 in the pathogenesis of Alzheimer's disease: is HIPK2 the link. Aging (Albany NY). 2010;2(9):545.

37. Uberti D, Lanni C, Racchi M, Govoni S, Memo M. Conformationally altered p53: a putative peripheral marker for Alzheimer's disease. Neurodegener Dis. 2008;5(3-4):209-11.

38. Le MT, Teh C, Shyh-Chang N, Xie H, Zhou B, Korzh V, et al. MicroRNA-125b is a novel negative regulator of p53. Gene Dev. 2009;23(7):862-76.

39. Hu Z, Yu D, Gu Qh, Yang Y, Tu K, Zhu J, et al. miR-191 and miR-135 are required for long-lasting spine remodelling associated with synaptic long-term depression. Nat Commun. 2014;5:3263.

40. Edbauer D, Neilson JR, Foster KA, Wang CF, Seeburg DP, Batterton MN, et al. Regulation of synaptic structure and function by FMRP-associated microRNAs miR-125b and miR-132. Neuron. 2010;65(3):373-84.

41. Terry RD, Masliah E, Salmon DP, Butters N, DeTeresa R, Hill R, et al. Physical basis of cognitive alterations in Alzheimer's disease: synapse loss is the major correlate of cognitive impairment. Ann Neurol. 1991;30(4): 572-80.

42. Selkoe DJ. Alzheimer's disease is a synaptic failure. Science. 2002;298(5594):789-91.

43. Scheff SW, Price DA, Schmitt FA, Mufson EJ. Hippocampal synaptic loss in early Alzheimer's disease and mild cognitive impairment. Neurobiol Aging. 2006;27(10):1372-84. 